\title{
Estudios ambientales del recurso hídrico en la región de Tandilia
}

\section{Environmental studies of water resources in the Tandilia region}

\author{
Víctor Alejandro Ruiz de Galarreta ${ }^{1}$, Corina Iris Rodríguez ${ }^{1,5}$, \\ Rosario Soledad Barranquero ${ }^{1,5}$, Marisol Cifuentes ${ }^{1,5}$, \\ Miguel Ángel Quiroga ${ }^{2}$, Roberto Landa², Anahí Tabera² ${ }^{2}$ Agustina Cortelezzi ${ }^{3,5}$, \\ Leandro Bogetti ${ }^{4}$ y Ariel Bidaure ${ }^{4}$
}

${ }^{1}$ Centro de Investigaciones y Estudios Ambientales (CINEA), Facultad de Ciencias Humanas (FCH), Universidad Nacional del Centro de la Provincia de Buenos Aires

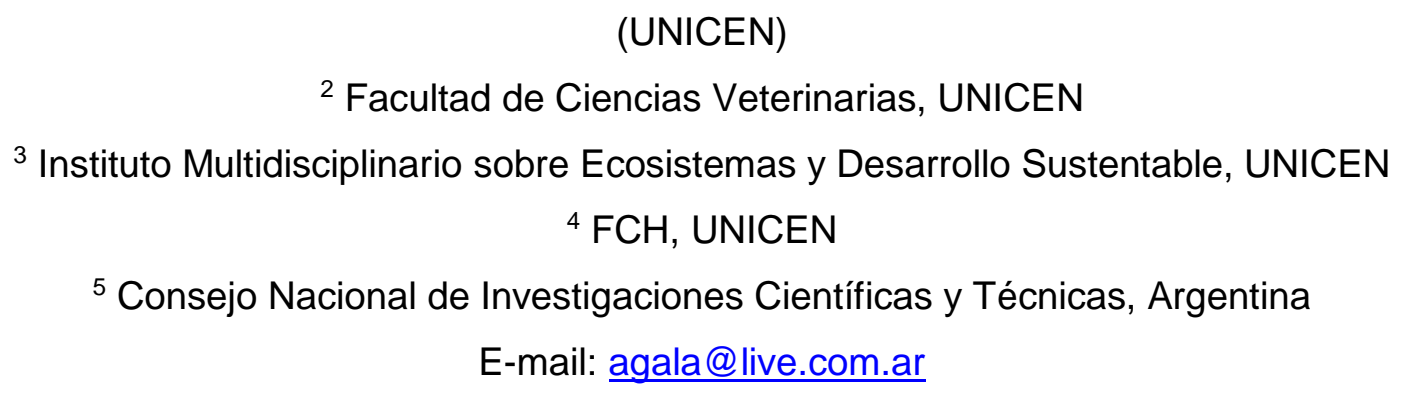

Ruiz de Galarreta, V. A., Rodríguez, C. I., Barranquero, R. S., Cifuentes, M., Quiroga, M. A., Landa, R., Tabera, A., Cortelezzi, A., Bogetti, L. \& Bidaure, A. (2017). Estudios ambientales del recurso hídrico en la región de Tandilia. Revista Estudios Ambientales, 5(2), 24-50. 


\section{RESUMEN}

Se presentan avances de estudios ambientales sobre el recurso hídrico en sectores serranos y periserranos de Tandilia. El objetivo general fue la profundización del análisis hidrológico ambiental en la región, basado en los antecedentes del grupo de investigación. Los objetivos específicos fueron: analizar la calidad, dinámica y gestión del recurso superficial; evaluar el manejo del agua subterránea en la región identificando sus principales limitaciones y potencialidades; y el análisis comparativo del sistema hídrico subterráneo en las cuencas de los arroyos Langueyú y Del Azul. Se parte de una visión integral, considerando al agua como un componente del sistema ambiental. Para cada objetivo se aplicaron metodologías específicas, que incluyeron técnicas y herramientas de las ciencias naturales y sociales. Los principales resultados mostraron las variaciones de caudal y calidad en el arroyo Langueyú desde su cabecera hacia aguas abajo, así como de la calidad del agua y del hábitat en el balneario del arroyo Chapaleofú en Rauch. Los avances en la caracterización hidrológica del embalse del Lago del Fuerte muestran la importancia del carácter efluente de sus tributarios y la afectación por vuelcos antrópicos. Tanto en la gestión del agua superficial en la cuenca del Langueyú como en el balneario de Rauch, uno de los principales problemas detectados es la escasa o nula participación de los usuarios del recurso. El análisis en sectores periurbanos y rurales identificó también esta debilidad, y coincidió en la falta de una visión integral y de una política institucional clara. Además, de la comparación de la hidrología subterránea en las cuencas del Langueyú y Del Azul se desprende que las principales diferencias se hallan en el manejo antrópico. Se concluyó que la gestión posee falencias en las diferentes escalas de trabajo analizadas, que repercuten en la calidad y dinámica de los recursos hídricos.

Palabras clave: hidrología regional, sistema ambiental, política institucional, participación, ordenamiento territorial.

\section{ABSTRACT}

The work shows the progresses achieved on the environmental studies carried out on the water resource, in hills and foothills sectors of Tandilia region. The main objective 
was to know in depth the environmental hydrological analysis in the region, based on the research group background studies. The specific objectives were: to analyze the quality, dynamic and management of surface resources; to evaluate the groundwater management in the region, identifying its main limitations and strengths; and the comparative analysis of the groundwater system in the Langueyú and Del Azul basins. The study starts from an integral approach, considering water as a component of the environmental system. For each objective, specific methodologies were applied, including techniques and tools of natural and social sciences. The main results showed the flow and quality variations in the Langueyú stream from its headwaters to downstream, as well as the water and habitat quality at the Chapaleofú recreation area in Rauch. The advances in the Lago del Fuerte hydrological characterization showed the importance of the relation between groundwater and surface water and the affectation by effluent discharges. Regarding to the management of surface water in the Langueyú basin and the recreation zone in Rauch, one of the main problems detected is the low or null participation by the resource's users. The peri-urban and rural sectors analysis also identified this weakness, and the lack of a systemic approach and a strong institutional policy. Moreover, the comparison of groundwater hydrology in the Langueyú and Del Azul basins shows that the main differences are found in human management. It was concluded that water management has shortcomings in the different work levels analyzed, which have an impact on water resources quality and dynamic.

Keywords: regional hydrology, environmental system, institutional policy, stakeholder participation, land use planning.

\section{INTRODUCCIÓN}

La complejidad del ambiente, en tanto confluencia de diversos elementos y procesos interrelacionados que funciona como una totalidad organizada, tiene una dinámica que debe ser estudiada desde una perspectiva sistémica (García, 2011). Respecto a los recursos hídricos, el enfoque más adecuado a esta perspectiva es el de la Gestión Integrada de los Recursos Hídricos (GIRH) como proceso pensado con un enfoque multisectorial donde se armonicen los diversos intereses de las poblaciones, con las 


\section{0 \\ revista estudios ambientales \\ publicación digital del CINEA

propias características y dinámica del entorno donde éstas habitan (Cap-Net, 2008; Global Water Partnership, 2009; Kennedy et al., 2009).

En la región del Sistema de Tandilia (Buenos Aires, Argentina) tienen sus nacientes distintas cuencas que drenan sus aguas desde el faldeo Norte serrano hacia la depresión de la cuenca del río Salado (Figura 1a). El presente estudio se llevó a cabo particularmente relacionado a tres de ellas (Langueyú, Chapaleofú y Del Azul) que cuentan con las poblaciones de Tandil, Rauch y Azul, respectivamente (Figura 1b). En dichas localidades se hace uso del recurso hídrico subterráneo para consumo humano y para la gran mayoría de las actividades antrópicas; en tanto, el recurso superficial es utilizado puntualmente con fines recreativos. En este contexto, se detectan demandas de diferentes sectores sociales por conocer aspectos relacionados a la problemática hídrica ambiental y de la salud a nivel local.

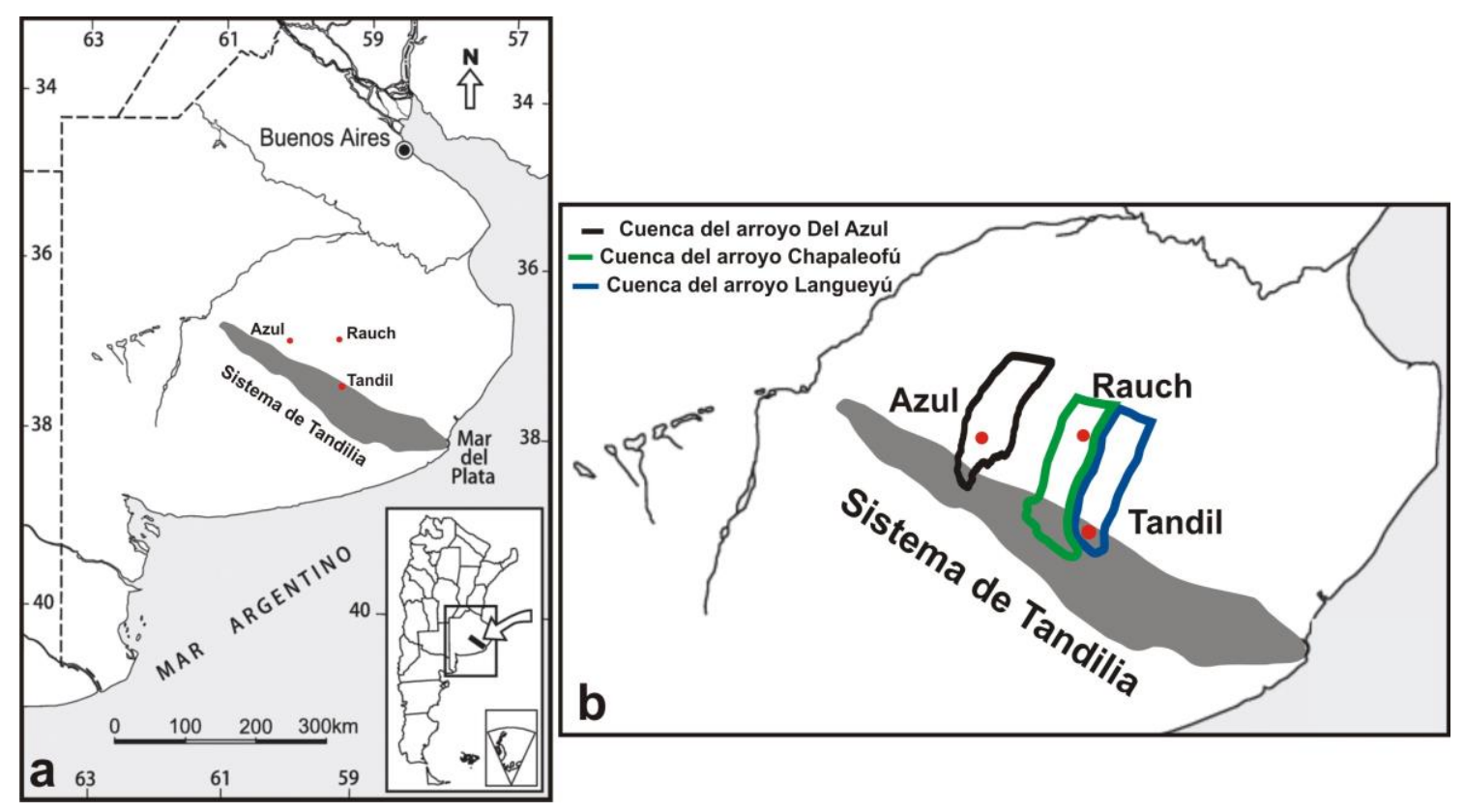

Figura 1. a) Ubicación del Sistema de Tandilia. b) Cuencas y localidades involucradas.

El trabajo se enmarca en el proyecto "Evaluación ambiental del recurso hídrico superficial y subterráneo en sectores serranos y periserranos de Tandilia", del Programa Nacional de Incentivos a Docentes Investigadores del Ministerio de Educación de la Nación (Código: 03/D283. Período 2015-2017). Dicho proyecto se elaboró considerando el estado de arte de la temática en la región, incluyendo los 
antecedentes específicos del grupo de trabajo, entre los que se destacan: Ruiz de Galarreta y Banda Noriega (2005), Ruiz de Galarreta (2006), Barranquero (2009 y 2015), Díaz (2010), Rodriguez (2010 y 2014), Rodriguez et al. (2010, 2011, 2013a y b), Ruiz de Galarreta et al. (2010 y 2011), Ruiz de Galarreta y Varni (2010), Pessolano (2011) y Barranquero et al. (2011, 2012, 2013 y 2014). La exploración de estos antecedentes permitió concluir que si bien se han generado avances tanto respecto a la evaluación de aspectos físicos del recurso hidrológico, como a la influencia antrópica sobre él, no se ha profundizado en la integración de los resultados de cara a la gestión y en la perspectiva regional.

Sumado a esto, si bien existen antecedentes en la región sobre los recursos hídricos superficiales como los estudios de IHLLA (1995; 2005a y 2005b) y Peluso et al. (1996), no se ha estudiado su vinculación con el recurso subterráneo y el abordaje ha sido con menor nivel de detalle como en Banda Noriega et al. (2008), Cifuentes (2012), Ruiz de Galarreta et al. (2013) y Barranquero et al. (2015).

Teniendo en cuenta entonces la vacancia temática se planteó como objetivo general del proyecto profundizar el análisis ambiental del recurso hídrico superficial y subterráneo en sectores serranos y periserranos del área de Tandilia. La investigación partió de los siguientes objetivos específicos:

A) Analizar la calidad, dinámica y gestión del recurso hídrico superficial en cuencas del faldeo norte del sistema de Tandilia, y determinar su aptitud para uso recreativo.

B) Evaluar comparativamente el sistema hídrico subterráneo en las cuencas de los arroyos Langueyú y Del Azul, con el propósito de elaborar un sistema de gestión ambiental del recurso.

C) Analizar el manejo del agua subterránea en la región periserrana de Tandilia e identificar las principales limitaciones y potencialidades que influyen en su gestión integrada.

\section{METODOLOGÍA}

El diseño de investigación ha incluido las siguientes actividades para lograr los objetivos específicos previamente mencionados: 


\section{Objetivo A}

A.1) Análisis de la dinámica y calidad del agua superficial en la cuencas de los arroyos Langueyú y Chapaleofú

Se realizaron recorridos trimestrales durante un año en la cuenca alta del arroyo Langueyú, considerando 11 sitios que incluyen (Figura 2): sus tributarios (Sitios 1 a 4), los dos afluentes principales del Langueyú luego de atravesar la ciudad entubados (Sitio 5 y 6), la zona de descargas de dos plantas de tratamiento cloacal y diversas industrias (Sitio 7 a 10), hasta finalizar aguas abajo de la ciudad (Sitio 11). Con la misma frecuencia, se realizaron aforos en 6 puntos para conocer sus variaciones naturales así como los posibles ingresos por vertidos antrópicos y se tomaron muestras de agua para la determinación de parámetros básicos de calidad del agua ( $\mathrm{pH}$, conductividad eléctrica y temperatura).

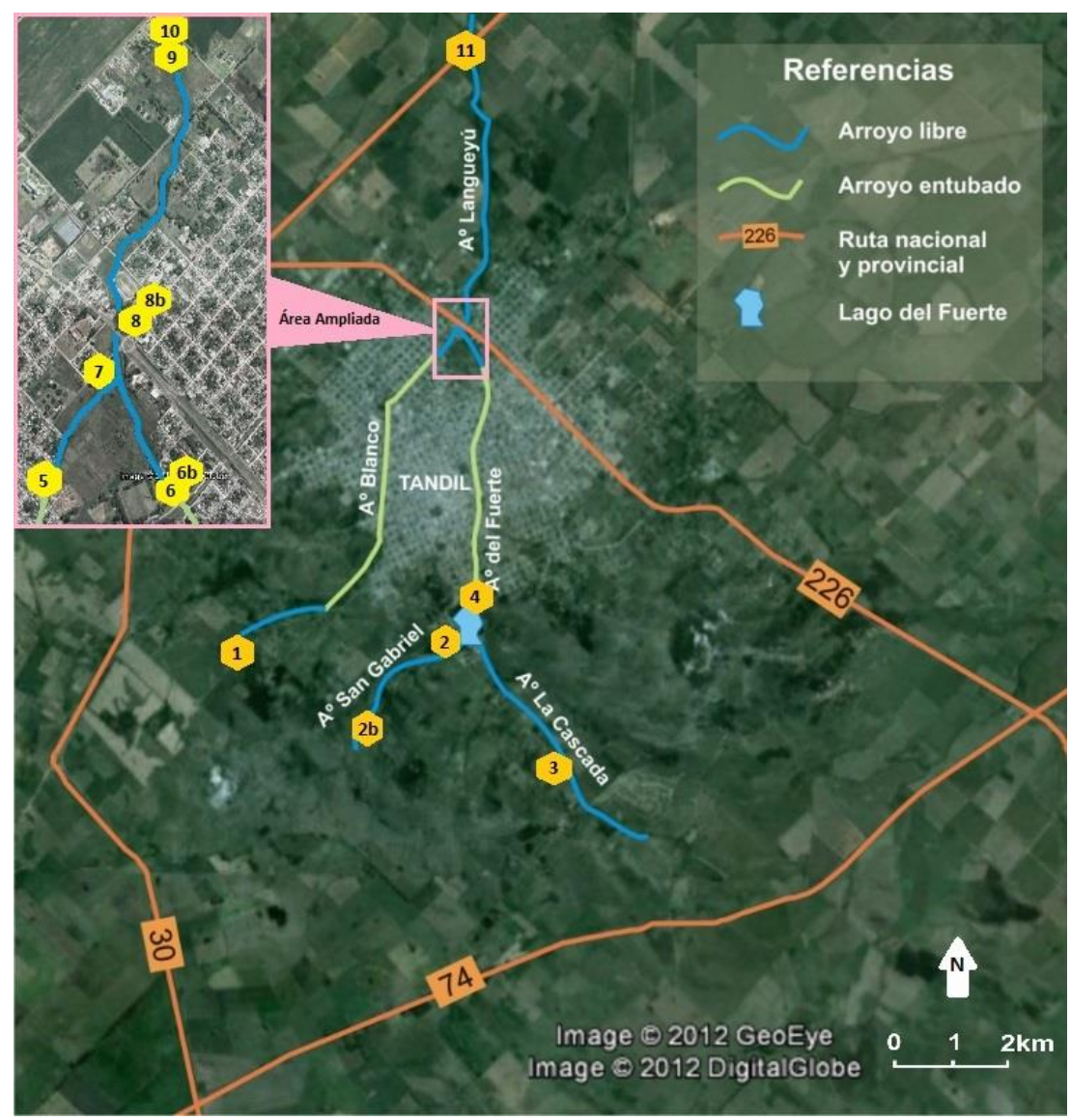

Figura 2. Cuenca alta arroyo Langueyú. Puntos de muestreos. Fuente: Bogetti (2015). 


\section{9 \\ revista estudios ambientales \\ publicación digital del CINEA

Por otro lado en el arroyo Chapaleofú, a partir de una tesina de grado (Bidaure, 2016), se seleccionaron 6 sitios de muestreo en el curso del arroyo en la localidad Rauch (Figura 3), distribuidos aguas arriba y abajo del Balneario Municipal, y en las propias instalaciones del espacio recreativo. Se llevaron a cabo dos campañas, una con anterioridad al inicio de las actividades de limpieza del balneario y la otra luego de la temporada recreativa. Se efectuó el muestreo para análisis fisicoquímicos (sólidos totales y suspendidos, conductividad, $\mathrm{pH}$, temperatura, oxígeno disuelto, cloruros, fósforo, nitratos, nitritos, amonio, $\mathrm{DBO}_{5}$ y $\mathrm{DQO}$ ), en cada punto se determinaron variables del hábitat físico (ancho del cauce, profundidad, velocidad de la corriente, caudal y cobertura vegetal). Para completar la caracterización del hábitat se aplicó un índice seleccionando los parámetros más representativos de los sistemas lóticos de la región pampeana (Barbour et al., 1999; Cortelezzi, 2010). Se colectaron muestras de sedimento para analizar el ensamble de macroinvertebrados bentónicos a partir de parámetros biológicos como el Índice Biótico para ríos y arroyos pampeanos (IBPAMP) de Rodrígues Capítulo et al. (2001). A través de la integración del análisis de calidad de agua, de hábitat físico y biota se definió la calidad ecológica. Por último, para determinar la calidad higiénica se efectuaron análisis microbiológicos.

Para todas las determinaciones de laboratorio, contempladas en cada una de las líneas de trabajo, se emplearon métodos analíticos normalizados correspondientes al APHA (2005).

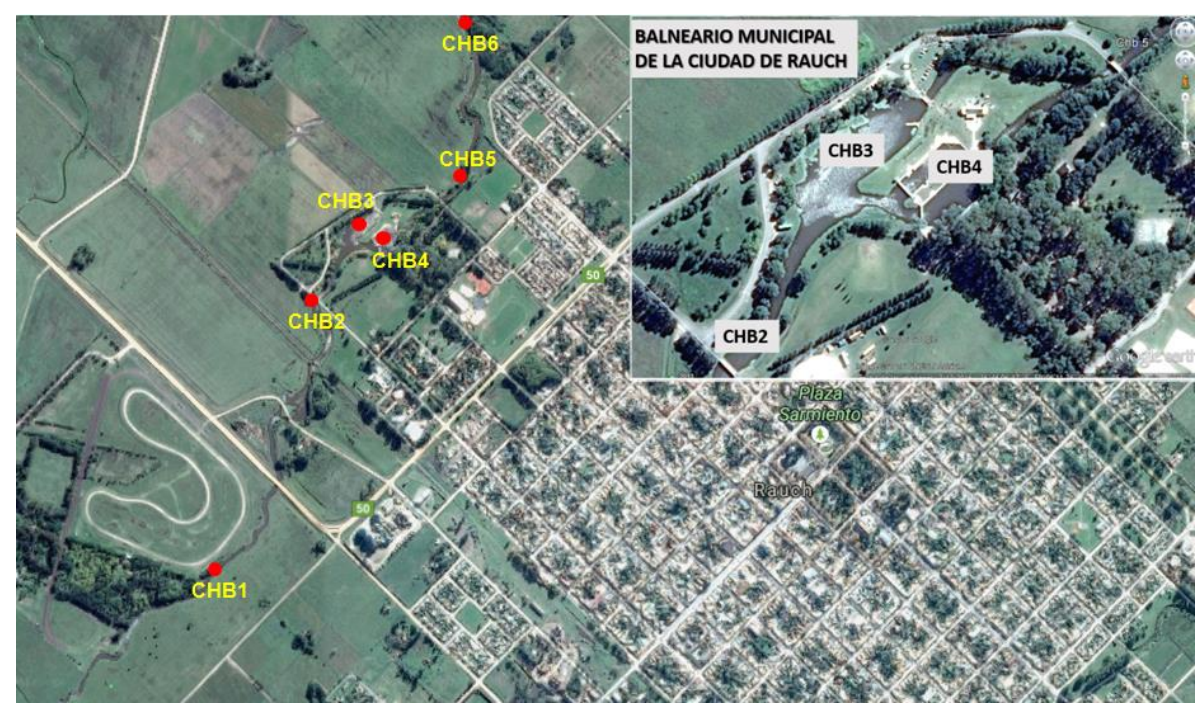

Figura 3. Balneario municipal de Rauch. Puntos de muestreo. Fuente: Bidaure et al. (2016). 


\section{A.2) Estudio ecohidrológico del embalse eutrófico del Lago del Fuerte}

Durante el período de una beca doctoral en curso de la Licenciada Marisol Cifuentes se ha planteado evaluar el estado hidrológico ambiental del embalse Lago del Fuerte de la ciudad de Tandil, teniendo en cuenta su cuenca de aporte, identificando las principales causas de eutrofización y desarrollando un modelo de eutrofización considerando el marco económico y social (Figura 4). Para ello se ha avanzado en la caracterización geológica, geomorfológica y climática de la cuenca de aporte de dicho embalse (2200 hectáreas) y en la determinación de la hidrodinámica e hidroquímica superficial desde las nacientes hasta la salida de las compuertas. Se realizaron muestreos en 4 puntos (arroyos San Gabriel y La Cascada, Lago del Fuerte y salida de la presa) durante un año con una frecuencia estacional.

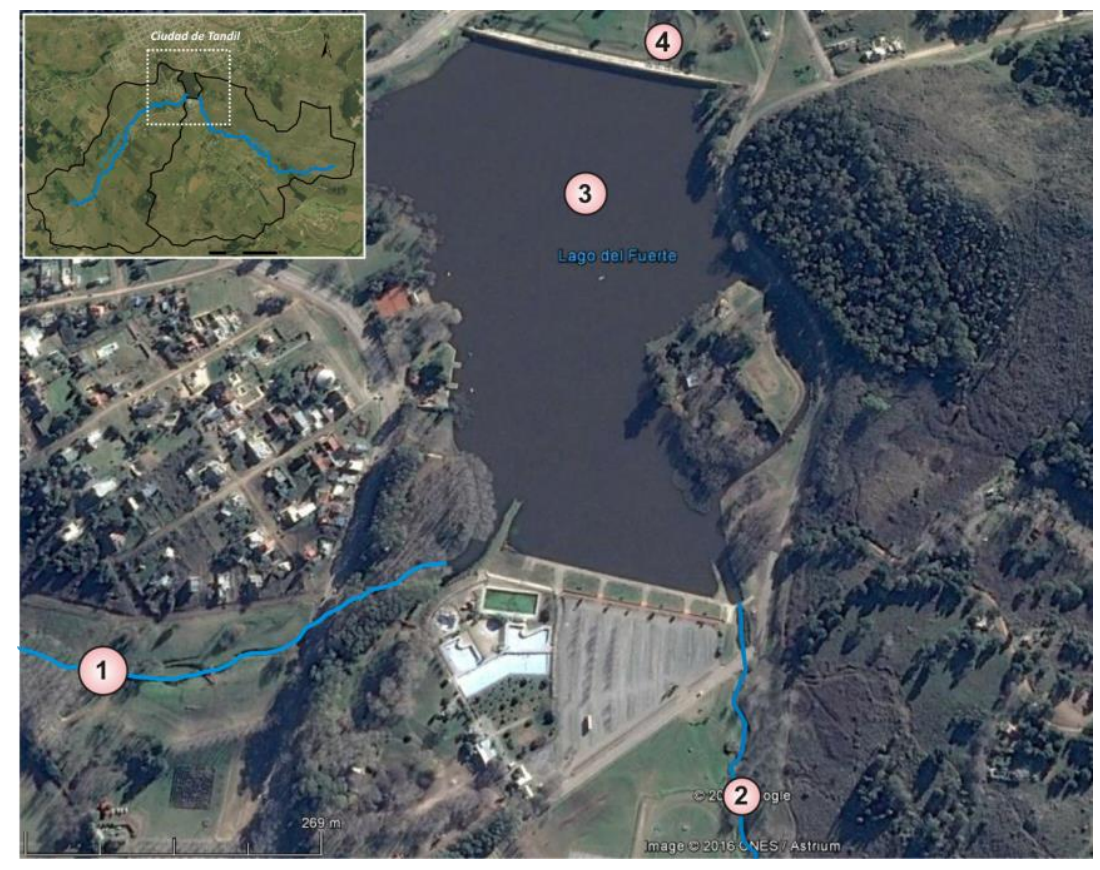

Figura 4. Lago del Fuerte Tandil. Puntos muestreo. Fuente: Cifuentes et al. (2017).

A.3) Evaluación del uso y gestión del recurso hídrico superficial en las cuencas de los arroyos Chapaleofú y Langueyú

En la cuenca del arroyo Langueyú se relevaron las opiniones, conocimientos y prácticas ambientales que la población de la ciudad de Tandil tiene con respecto a dicho arroyo y sus tributarios, a través de encuestas y entrevistas. Se realizaron 128 encuestas estructuradas a habitantes de distintos sectores de la ciudad, que 
incluyeron tres zonas: pre-entubado (ZPre), entubado (ZE), post-entubado (ZPost), considerando que representan distintos entornos por su posición relativa a los arroyos. Se realizaron tres entrevistas no estructuradas a informantes clave: un referente barrial y un trabajador social de la zona post-entubado, y un vecino que reside en cercanías del arroyo.

Para analizar la gestión ambiental del Balneario de la ciudad de Rauch ubicado sobre el arroyo Chapaleofú, Bidaure (2016) consideró la base teórica-metodológica del ciclo de planificación GIRH (GWP, 2009). De acuerdo a ello se definieron como ejes transversales: a) la política institucional; b) el diagnóstico; y c) la disponibilidad de información ambiental. Se realizaron encuestas y entrevistas a informantes clave y se consideró información secundaria (informes y registros del balneario y del Municipio de Rauch). Se efectuaron encuestas intencionales no probabilísticas a alumnos de escuelas primarias y secundarias de la localidad, con un total de 164 y 162 casos respectivamente, a fin de conocer el uso que realizan del Balneario y la percepción que poseen acerca del espacio recreativo y de la calidad del agua. En tanto, la modalidad de las entrevistas fue de tipo no estructurada focalizada, con preguntas abiertas, a actores involucrados en la gestión del balneario.

\section{Objetivo $B$}

B.1) Evaluación comparativa del sistema hídrico subterráneo en las cuencas de los arroyos Langueyú y Del Azul

Se llevó a cabo la recopilación y análisis de los estudios antecedentes de las cuencas de los arroyos Langueyú y Del Azul. A partir de esta información se describieron las similitudes y diferencias generales entre las cuencas utilizando los principios del grado de analogía de conceptos de Duverger (1981). Se realizó un primer avance en la comparación considerando: litología, hidrodinámica, hidroquímica e influencia antrópica; a través de mediciones de niveles freáticos y análisis fisicoquímicos en redes de monitoreo del agua subterránea e impactos potenciales al sistema según las características del medio físico y las actividades antrópicas (Barranquero et al., 2016a). Este trabajo dio el marco para definir las variables involucradas en el sistema. Se definieron las técnicas de comparación de las variables hidrogeológicas: a) hidrolitología (ambientes hidrolitológicos, tipo de acuífero, y profundidad del basamento cristalino); b) hidrodinámica (recarga, profundidad del agua subterránea, 
sitios de descarga, y relación agua superficial-agua subterránea); y c) hidroquímica (tipificación, procesos hidrogeoquímicos fundamentales, y calidad del agua subterránea para distintos usos). Específicamente a través de una tesina de grado se están definiendo las técnicas de comparación de las variables referidas a la relación de la población con el recurso hídrico y las políticas públicas vinculadas al mismo.

En el aspecto que más se ha avanzado con la comparación es en el hidroquímico, realizando una interpretación conjunta de bases de datos de perforaciones en ambas cuencas a través de análisis estadístico descriptivo, MA-PCA y N-PCA (Barranquero et al., 2016b).

\section{Objetivo C}

C.1) Análisis del manejo del agua subterránea en sectores periurbanos y rurales de la región periserrana de Tandilia.

Se seleccionaron casos de estudio dentro del partido de Tandil, incluyendo dos sectores periurbanos (barrios Cerro Los Leones y Don Bosco) y dos localidades rurales (Gardey y María Ignacia), debido al interés que presentan por su ausencia o cobertura parcial de servicios sanitarios. Se monitoreó la hidrodinámica subterránea y la calidad del agua para consumo, mediante la determinación del contenido de nitratos y análisis microbiológicos. Asimismo, se describieron las características constructivas y de diseño de los pozos de extracción.

C.2) Identificación de las principales limitaciones y potencialidades que influyen en el manejo integrado del agua subterránea en el área

Se evaluaron las potencialidades y limitaciones del manejo integrado del agua subterránea en ambientes periserranos de Tandilia, mediante la metodología de la matriz DAFO (Debilidades, Amenazas, Fortalezas y Oportunidades) que constituye la base para el ordenamiento territorial (Pujadas y Font, 1998; Gómez Orea y Gómez Villarino, 2012). Este tipo de análisis permite detectar las oportunidades que son aprovechables, las debilidades que deben superarse, y las amenazas que pueden ser contrarrestables. En ese sentido, la integración generada por esta metodología resulta útil para el diseño de indicadores de seguimiento y facilita la elaboración de estrategias de gestión sostenible. 


\section{RESULTADOS Y DISCUSIÓN}

A partir de la profundización de las diferentes líneas de investigación que se muestra en este trabajo, se continuó ampliando el conocimiento del sistema hídrico ambiental en la región, con énfasis en los sectores serranos y periserranos del cordón serrano de Tandilia. A continuación se detallan los resultados correspondientes a cada objetivo específico.

\section{Objetivo A}

A.1) Análisis de la dinámica y calidad del agua superficial en la cuencas de los arroyos Langueyú y Chapaleofú

En la cuenca del arroyo Langueyú, las mediciones realizadas por Bogetti (2015) presentadas en la Tabla 1 han determinado que el caudal se incrementó notablemente aguas abajo. Los tributarios son naturalmente efluentes y además reciben efluentes pluviales, industriales y cloacales que acentúan esa crecida. Se verificó en todas las fechas de muestreo un aumento brusco de caudal en los Sitios 7 y 9 , zona donde el arroyo Langueyú recibe aportes antrópicos, fundamentalmente debido a la descarga de dos plantas de tratamiento cloacal.

Con respecto a la calidad del agua, la Tabla 1 indica que la conductividad eléctrica aumenta aguas abajo debido a un componente acumulativo de los principales aportes de efluentes industriales y cloacales en las zonas del arroyo entubado (Sitios 5 y 6 ) y post-entubado (Sitios 7 a 11). Los valores de temperatura también aumentan hacia aguas abajo. Se detectó una elevación puntual en la desembocadura del lago Del Fuerte (Sitio 4) que estaría provocado por la influencia de la radiación solar sobre el embalse. El incremento progresivo en el sentido de escurrimiento se debe a que el agua que transportan los tributarios en cabeceras corresponde al caudal subterráneo básico, mientras que hacia aguas abajo se verifica la influencia de efluentes antrópicos con temperaturas superiores.

En el sector estudiado del arroyo Chapaleofú, Bidaure (2016) determinó una calidad de agua buena a muy buena de acuerdo a los parámetros fisicoquímicos. Respecto a las variables de hábitat físico, el ancho del arroyo fue lo más alterado en el balneario. A diferencia de otros arroyos pampeanos, la cobertura vegetal fue muy baja en todo el tramo de estudio, disminuyendo por tanto la diversidad de los hábitats funcionales. El IBPAMP (Figura 5) denotó un fuerte impacto en las instalaciones del balneario (sitios 
CHB2, CHB3 y CHB4), aunque el análisis en conjunto de la biota en el resto del cauce permitió definir una calidad alta de la misma, con un gran número de taxa bien representados y diversos. La mayor densidad corresponde al grupo oligoquetos, gusanos redondos que dominan los sedimentos de la mayoría de los cuerpos de agua pampeanos (Figura 6). El sitio correspondiente a la laguna de contención temporaria del balneario se diferenció por no responder al incremento de población propio del ciclo estacional, por lo que la disminución de la velocidad del agua y su estancamiento temporario en este sitio podría ser una fuente de contaminación para el resto del sistema (Bidaure et al., 2016).

Tabla 1. Valores medios para las 4 estaciones del año consideradas. Fuente: Bogetti (2015).

\begin{tabular}{|c|c|c|c|}
\hline Sitios & $\begin{array}{c}\text { Caudal } \\
\left(\mathrm{m}^{3} / \mathrm{seg}\right)\end{array}$ & $\begin{array}{c}\text { Conductividad } \\
\text { eléctrica } \\
(\mu \mathrm{S} / \mathrm{cm})\end{array}$ & $\begin{array}{c}\text { Temperatura } \\
\left({ }^{\circ} \mathrm{C}\right)\end{array}$ \\
\hline 1 & 0,058 & 638 & 16,9 \\
\hline 2 & 0,050 & 240 & 16,0 \\
\hline 3 & --- & 92 & 18,7 \\
\hline 4 & --- & 228 & 19,4 \\
\hline 5 & 0,200 & 682 & 16,9 \\
\hline 6 & 0,159 & 486 & 17,7 \\
\hline 7 & 0,345 & 528 & 18,4 \\
\hline 8 & --- & 680 & 19,3 \\
\hline 9 & 0,871 & 920 & 20,6 \\
\hline 10 & --- & 876 & 19,6 \\
\hline 11 & --- & 858 & 20,6 \\
\hline
\end{tabular}




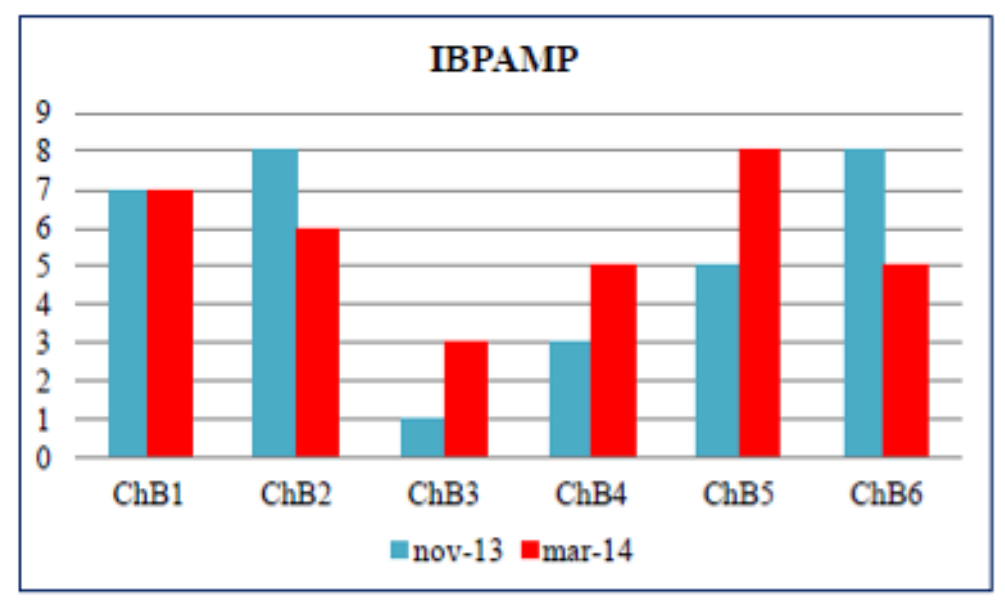

Figura 5. Valores del índice biótico pampeano en las seis estaciones de muestreo para ambos períodos. Fuente: Bidaure (2016).
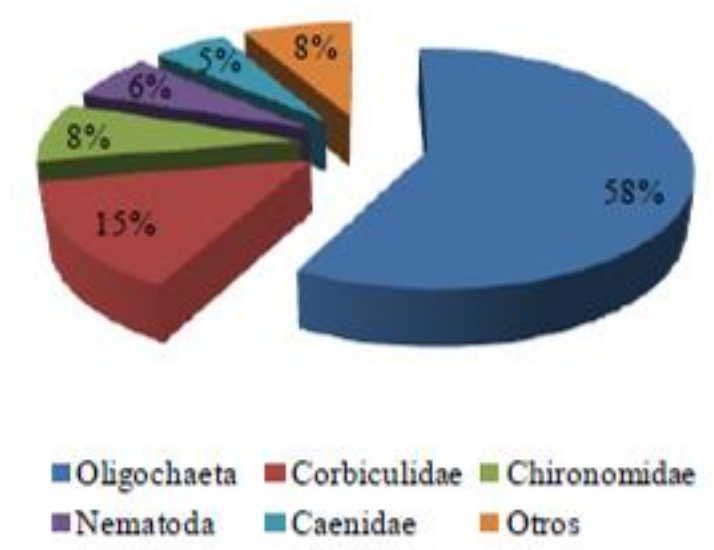

Figura 6. Porcentaje de macroinvertebrados encontrados en ambas campañas de muestreo (2013-2014) en el tramo analizado. Fuente: Bidaure (2016).

En cuanto a la calidad higiénica, se detectó la presencia de esporas de Clostridios sulfito reductores y de Escherichia coli en todos los sitios. Estos resultados, combinados con una concentración importante de Coliformes totales en los sitios propios del balneario, indican que es necesario implementar un monitoreo sistemático de la calidad higiénica, al menos previo a cada temporada estival de uso intensivo.

\section{A.2) Estudio ecohidrológico del embalse eutrófico Lago del Fuerte}

Según los resultados de Cifuentes et al. (2017), y como puede verse en la Figura 7, en 


\section{a \\ revista estudios ambientales

publicación digital del CINEA

lo que respecta a la caracterización fisicoquímica de las aguas superficiales la conductividad en los dos tributarios presentó valores más elevados que el lago y la salida en casi todas las estaciones del año, excepto en invierno. A su vez los valores del lago y la salida fueron similares durante todo el período de estudio.

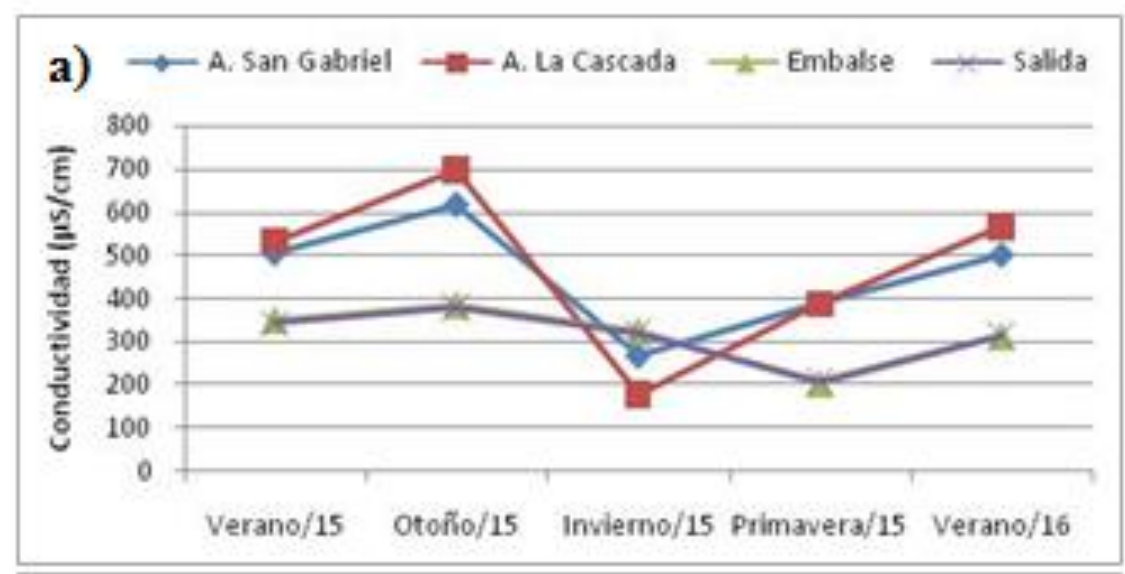

b) $\rightarrow$ A. san Gabriel $\rightarrow$ - A. La Cascada $\rightarrow$ Embalse $\rightarrow$ salida
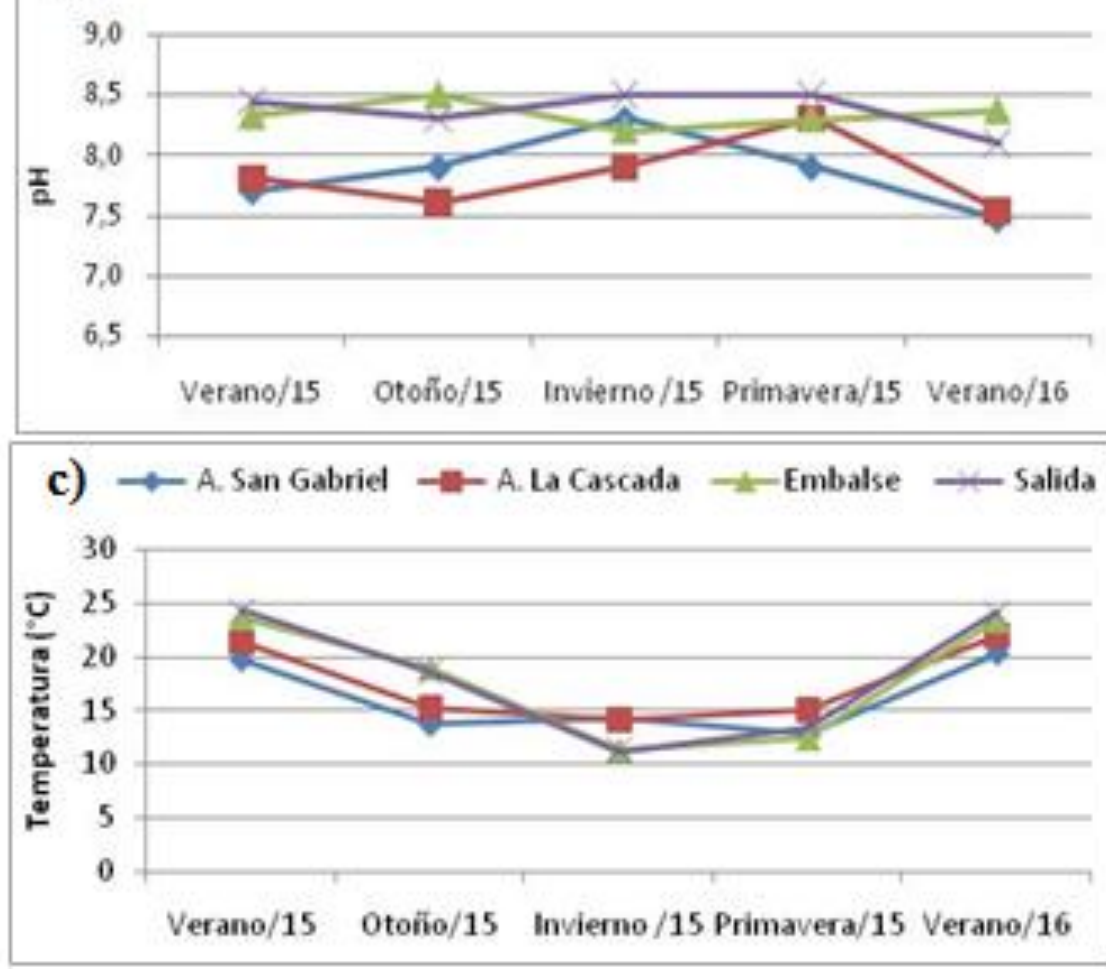

Figura 7. Valores estacionales en los sitos de muestreos. a) Conductividad. b) pH

c) Temperatura. Fuente: Cifuentes et al. (2017). 
En todos los puntos de muestreo los mayores registros de conductividad se dieron en otoño. Esto coincide con un período de escasas precipitaciones y un marcado déficit hídrico lo que estaría asociado a un aumento de la salinidad en el embalse por una disminución en la tasa de renovación, así como por el aporte de los arroyos con un bajo caudal, conformado principalmente por el caudal básico subterráneo.

Los registros de $\mathrm{pH}$ fueron levemente mayores en el embalse que en los arroyos, principalmente en verano y otoño. La diferencia entre los sitios podría deberse a procesos de fotosíntesis en el embalse.

A la salida del lago en verano y otoño se generó un incremento de la temperatura en promedio de $4^{\circ} \mathrm{C}$ con respecto al agua que ingresa por los arroyos, mientras que en invierno se registró un descenso de $3^{\circ} \mathrm{C}$. Estas diferencias de temperatura estarían indicando el efecto térmico que genera la presa.

Se considera que las diferencias temporales y espaciales de los parámetros analizados se debieron al aporte de aguas subterráneas en los arroyos, la precipitación directa y el escurrimiento superficial en el lago, así como a la escasa profundidad, exposición a la radiación, y tiempo de residencia en este último.

Con el propósito de alcanzar el estudio ecohidrológico del embalse actualmente se está trabajando en la caracterización de las cargas contaminantes que impactan al lago, las estimaciones de los balances hidrológicos y de nutrientes, así como el análisis cuali-cuantitativo del fitoplancton, enfocándose principalmente en la presencia de cianobacterias. Con ello se espera determinar el período del año en que la abundancia de estas algas reviste riesgo sanitario para la población usuaria, afectando su aptitud recreativa.

A.3) Evaluación del uso y gestión del recurso hídrico superficial en las cuencas de los arroyos Chapaleofú y Langueyú

De acuerdo al estudio social efectuado por Bogetti (2015), en el caso del arroyo Langueyú se obtuvo que el nivel de conocimiento de la población sobre los arroyos de Tandil es bajo. Sólo una minoría de los encuestados tiene noción de que existe más de un arroyo en la ciudad y desconoce su denominación, y muy pocos han tenido acceso a datos científicos sobre la calidad de sus aguas. Además, la mayoría no sabe qué tipos de vertidos reciben los arroyos. Se encontró que no existen canales o 


\section{0 \\ revista estudios ambientales \\ publicación digital del CINEA

mecanismos públicos de difusión de información respecto al sistema hídrico superficial o, de existir, tienen un impacto menor en la población.

En cuanto a las prácticas, es muy baja la participación ciudadana directa en asambleas o grupos que traten temas relativos a los arroyos y en reclamos formales a autoridades públicas. Posiblemente, el escaso conocimiento incide en el bajo uso directo del arroyo para actividades de baño, pesca o recreo.

En lo que respecta a la opinión, la Tabla 2 refleja que la mayoría considera que el estado de sanidad de los arroyos de Tandil es de regular a muy malo. Las principales causas que los encuestados atribuyen son: irresponsabilidad ciudadana (22\%), vertidos de empresas (17\%), gestión pública deficiente (15\%), desinterés de las autoridades en el tema (14\%) y falta de educación (13\%). En las zonas donde existen arroyos en superficie, y principalmente en la zona post-entubado (ZPost), los vecinos aprecian en mayor grado su degradación ambiental.

Tabla 2. Opiniones sobre el estado de sanidad de los arroyos según zonas.

Fuente: Bogetti (2015).

\begin{tabular}{|c|c|c|c|c|c|c|}
\hline Zona & $\begin{array}{c}\text { Muy } \\
\text { bueno }\end{array}$ & Bueno & Regular & Malo & $\begin{array}{c}\text { Muy } \\
\text { malo }\end{array}$ & $\begin{array}{c}\text { Sin } \\
\text { respuesta }\end{array}$ \\
\hline ZPre & $0 \%$ & $12,5 \%$ & $33,3 \%$ & $25 \%$ & $20,8 \%$ & $8,3 \%$ \\
\hline ZE & $3,3 \%$ & $13,1 \%$ & $27,9 \%$ & $18 \%$ & $11,5 \%$ & $26,2 \%$ \\
\hline ZPost & $0 \%$ & $11,1 \%$ & $27,7 \%$ & $11,1 \%$ & $27,7 \%$ & $22,2 \%$ \\
\hline
\end{tabular}

El análisis de la gestión ambiental del arroyo Chapaleofú en el Balneario Municipal de Rauch arrojó que no cuenta con una política institucional, con lo cual no es posible identificar su visión política, ni el compromiso de gobierno y la coordinación del equipo interviniente para llevarla adelante (Bidaure, 2016).

La definición de roles y la participación de actores estuvo en concordancia con la ausencia de política institucional, ya que se superponen distintas dependencias de la Municipalidad de Rauch ejerciendo roles importantes en cuanto al manejo del balneario pero sin una buena comunicación ni coordinación entre ellos. Sumado a esto, tanto la Dirección de Ambiente como el área de salud tienen una participación muy limitada, a pesar que los antecedentes de gestión de recursos hídricos han 
demostrado que son ejes fundamentales (Custodio et al., 2008; Gutiérrez, 2010). Se detectó además que los usuarios del espacio recreativo no tienen canales de participación en las decisiones de gestión.

El manejo del balneario no incluye el diagnóstico, que debería ser continuo para retroalimentar la política institucional (GWP, 2009). No se realizan análisis para monitorear la calidad del agua, lo cual es crítico considerando los riesgos a la salud que implica el uso con contacto directo, y la concurrencia mayoritaria en familia para la natación como actividad preponderante (Figura 8).
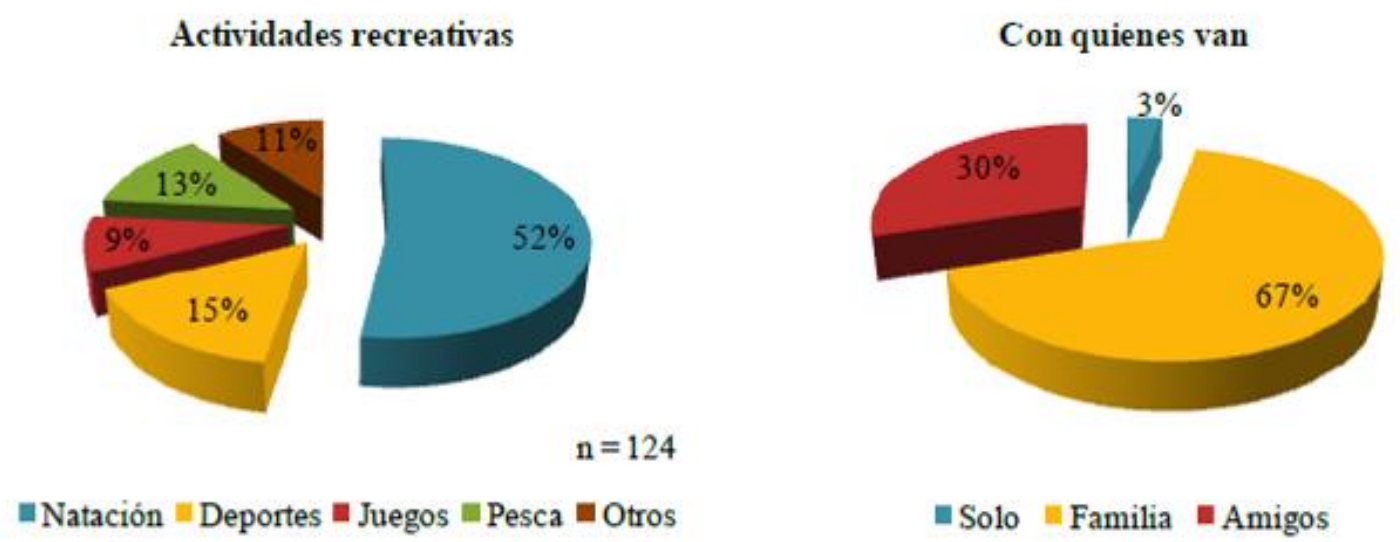

Figura 8. Actividades recreativas desarrolladas en el predio del Balneario Municipal y compañía con la que concurren al mismo. Fuente: Bidaure (2016).

En cuanto a la disponibilidad de información ambiental, se identificó escasa documentación y comunicación interna, y una comunicación externa prácticamente nula. No se detectó una programación de estrategias e instancias para llevar adelante la documentación, comunicación y difusión de esta información.

\section{Objetivo $B$}

B.1) Evaluación comparativa del sistema hídrico subterráneo en las cuencas de los arroyos Langueyú y Del Azul

De acuerdo a los aspectos definidos para su comparación (litología, hidrodinámica, hidroquímica e influencia antrópica), las cuencas de los arroyos Langueyú y Del Azul presentaron más similitudes que diferencias. Los ambientes hidrogeológicos que 


\section{0 \\ revista estudios ambientales \\ publicación digital del CINEA

definen la disponibilidad de agua subterránea (fisurado y poroso) y el régimen climático, en líneas generales, así como el total de los excesos y déficits y sus meses de ocurrencia en el balance hídrico (Figura 9), fueron las principales similitudes. Se identificaron diferencias respecto al emplazamiento de cada ciudad principal en la cuenca y el aprovechamiento de los recursos hídricos, así como en la dimensión y particularidades del potencial de contaminación producto del manejo antrópico.

Se observa que las cuencas poseen en líneas generales el mismo comportamiento hidroquímico, siendo comunes los procesos fundamentales que explican sus variaciones temporales: a) el aumento de la salinidad en la dirección del flujo subterráneo; b) el intercambio iónico calcio-magnesio por sodio en la misma dirección; $y, c)$ la contaminación, a partir de distintas fuentes en cada cuenca, con nitratos de tipo puntual y multipuntual (Barranquero et al., 2016 a y b).
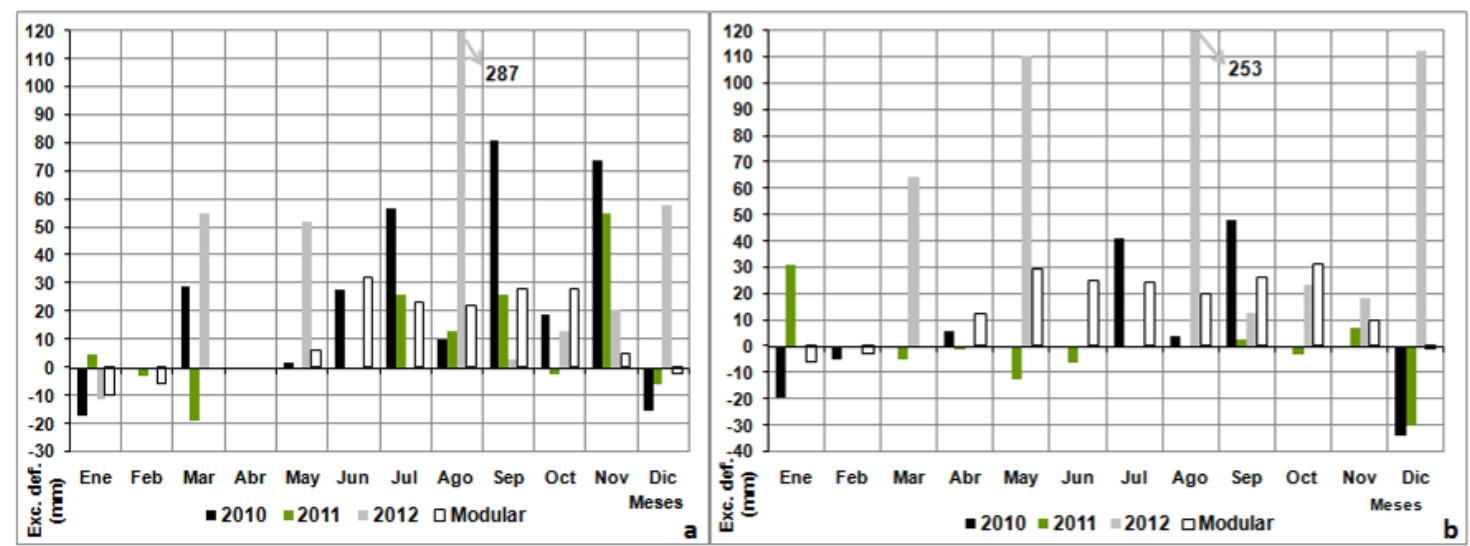

Figura 9. Comparación de excesos y déficits entre el balance hídrico modular y los años 2010, 2011 y 2012. a) Tandil. b) Azul. Fuente: Barranquero et al. (2016a).

La comparación permitió definir que las cuencas presentan diferencias más relevantes en cuanto al uso y la gestión del recurso que en sus características naturales, razón por la cual a nivel regional es importante profundizar en estos aspectos como insumo para la elaboración de estrategias para la gestión integral de los recursos hídricos. 


\section{Objetivo $C$}

C.1) Análisis del manejo del agua subterránea en sectores periurbanos y rurales de la región periserrana de Tandillia.

Los cuatro sectores de estudio seleccionados se compararon a partir del análisis de las siguientes variables centrales: modo de abastecimiento de agua, cualidades socioeconómicas más relevantes, características de las perforaciones y sus problemas para la extracción, calidad del recurso y los principales focos contaminantes.

La problemática hídrica en estos sectores tiene causas comunes, determinadas por la cobertura parcial o ausencia de servicios sanitarios y el escaso conocimiento sobre el funcionamiento del sistema hídrico subterráneo. Dichas causas, sumadas a la falta de aplicación de normas de seguridad ambiental para las captaciones de agua y los sistemas de descarga de efluentes domiciliarios, determinan la contaminación del agua a escala local mediante una vinculación hidrodinámica entre perforaciones y sitios de vertido de efluentes. Los contenidos de nitratos y los microorganismos patógenos que exceden lo recomendado por la legislación argentina evidencian esta situación. Además, se suman otros aspectos como las características constructivas de las perforaciones que no garantizan su protección sanitaria y la presencia de cargas contaminantes en las cercanías de las fuentes de agua.

Los problemas detectados ocurren independientemente del posicionamiento socioeconómico de la población (Rodriguez y Jacinto, 2017). La principal diferencia radica en que los sectores de mejor nivel socioeconómico pueden realizar nuevas y mejores perforaciones así como poseer cañerías y tanques de almacenamiento en buen estado, e incluso adquirir agua potable envasada para el consumo humano.

C.2) Identificación de las principales limitaciones y potencialidades que influyen en el manejo integrado del agua subterránea en el área

El análisis DAFO permitió identificar las fortalezas y debilidades internas referidas al manejo del agua subterránea y las oportunidades y amenazas provenientes del ámbito externo (Rodríguez, 2014).

Entre las fortalezas, las movilizaciones sociales en relación al agua contrarrestan la escasa participación de los actores locales en la temática, y contribuye a la generación de conciencia ciudadana sobre la necesidad de abastecer de servicios sanitarios. Otra fortaleza es la existencia de grupos de investigación de la UNICEN enfocados al 
diagnóstico del sistema hidrológico y al ordenamiento territorial. Esta fortaleza debiera ser aprovechada por los tomadores de decisiones para desarrollar una adecuada planificación territorial; orientando por ejemplo las infraestructuras públicas hacia el abastecimiento de servicios sanitarios básicos en forma previa al desarrollo urbano.

La ausencia de una mirada sistémica en dicha planificación se constituye en una debilidad, cuya superación requiere trabajar con una multiplicidad de organismos gubernamentales vinculados a la temática y lograr la participación y actuación conjunta de actores a nivel local, provincial y nacional.

El desarrollo de la actividad turística se presenta, por un lado, como una amenaza externa que fomenta la edificación en zonas serranas, pero además constituye una oportunidad porque pone en valor los recursos naturales.

Se destaca como una de las principales oportunidades la existencia de legislación que rige sobre distintos aspectos hidrológicos, que sin embargo requiere trabajar en su aplicación y control. Otra oportunidad es la Ley Provincial de Paisaje Protegido № 14.126/2010 que puede contribuir a superar la ausencia de planificación de los usos del suelo y la preservación del recurso hídrico, considerando especialmente que dicha área abarca toda la ciudad de Tandil y coincide con las cabeceras de tres cuencas.

\section{CONCLUSIONES}

Los estudios ambientales sobre los recursos hídricos de la región de Tandilia llevados adelante por el grupo de trabajo han permitido identificar las principales problemáticas relacionadas al agua superficial y subterránea en el área de estudio, en pos del análisis ambiental de la temática hidrológica regional.

La complejidad del sistema ambiental hace necesario el uso de distintas metodologías y herramientas, tanto de las ciencias sociales como naturales, para avanzar en la caracterización del recurso agua y la comprensión integral de su gestión.

Se ha logrado una caracterización detallada del sistema hídrico, tanto de su calidad para distintos usos, como de su dinámica y también la afectación por parte de actividades humanas. Asimismo, se ha avanzando en el análisis de la gestión actual del agua, integrando el recurso superficial con el subterráneo a nivel de las cuencas del faldeo norte de las sierras de Tandilia. Se han tenido en cuenta los actores involucrados en dicha gestión así como las prácticas individuales de los habitantes y las políticas públicas por parte del Estado en sus distintos niveles. 
Se concluye que la gestión posee falencias en las diferentes escalas de trabajo analizadas, que repercuten en la calidad y dinámica de los recursos hídricos.

Se evidencia la falta de una visión integral y de una política institucional clara que se encargue del ordenamiento de los usos del suelo y servicios sanitarios, así como del seguimiento periódico de las características del agua, tanto subterránea como superficial, que permita formular y rectificar las políticas, optimizando recursos y resultados. Todo ello redunda en la ausencia de información, tanto entre las diferentes dependencias del Estado así como hacia la sociedad.

Ante este contexto, se ha avanzado en la elaboración de pautas de gestión y transferencia que favorecen los procesos de toma de decisión y estimulan el alcance de los objetivos del desarrollo sustentable. La producción de esta información de base y de herramientas de gestión ambiental constituye un insumo de aplicación directa en la toma de decisiones a escala local y regional. De igual modo, es necesario continuar investigando en diversos aspectos que se vinculan a la problemática hidrológica y ambiental regional.

\section{AGRADECIMIENTOS}

Los análisis de calidad del agua fueron realizados en el Laboratorio de Análisis Bioquímicos y Minerales (LAByM) y el Laboratorio de Calidad de los Alimentos, ambos de la Facultad de Ciencias Veterinarias de la UNICEN, e Instituto de Limnología "Dr. Raúl A. Ringuelet" (CONICET-UNLP).

\section{BIBLIOGRAFÍA}

APHA. (2005). Standard methods for examination of water and wastewater. 21 th ed. Washington DC: American Public Health Association, American Water Works Association and Water Pollution Control Federation.

Barbour, M.T., J. Gerritsen, B.D. Snyder y J.B. Stribling. (1999). Rapid bioassessment protocols for use in streams and wadeable rivers: Periphyton, benthic macroinvertebrates, and fish. Second Edition. Washington, D.C: U.S. Environmental Protection Agency.

Banda Noriega, R; Ruiz de Galarreta, A; Barranquero, R; Díaz, A; Miguel, E; y Rodríguez, C. I. (2008). Caracterización de cargas contaminantes generadas 
en Tandil y su implicancia en el recurso hídrico. Buenos Aires, Argentina. En: Yaglián, E. y Rodríguez, M. E. (Eds.). I/ Congreso Internacional sobre gestión y tratamiento integral del agua (pp. 451- 463). Córdoba, Argentina: Fundación ProDTI.

Barranquero, R. (2009). Análisis hidrogeológico y evaluación de cargas contaminantes en la cuenca del arroyo Langueyú (Tesis de Maestría en Ciencias Hídricas). Universidad Nacional de La Pampa, Santa Rosa.

Barranquero, R., Varni, M., Ruiz de Galarreta, V. A. y Ocampo D. (2011). Evaluación del comportamiento hidrodinámico y cuantificación de la recarga en la cuenca del arroyo Langueyú, partido de Tandil. Revista de Geología Aplicada a la Ingeniería y al Ambiente, 27: 63-72.

Barranquero, R., Varni, M., Ruiz de Galarreta, V. A. y Banda Noriega, R. (2012). Aporte de la hidroquímica al modelo conceptual del sistema hídrico subterráneo. Tandil, Argentina. GEOACTA 37 (2): 130-146.

Barranquero, R., Varni, M., Ruiz de Galarreta, Quiroga, M. y Landa, R. (2013). Assessment of the hydrochemical characteristics of the Langueyú creek basin applying multivariate statistical analysis. The Journal of the Argentine Chemical Society, Vol. 100, 9-24.

Barranquero, R., Pardo, R., Varni, M., Ruiz de Galarreta, A. y Vega, M. (2014). Modelling of the groundwater hydrological behaviour of the Langueyú creek basin by using N-way multivariate method. Hydrological Processes 28 (17): 4743-4755.

Barranquero, R. (2015). Análisis y evaluación del sistema hidrogeológico ambiental en la cuenca del arroyo Langueyú, Tandil, Buenos Aires. (Tesis de doctorado). Universidad Nacional de Córdoba, Córdoba.

Barranquero, R. S., Varni, M. y Ruiz de Galarreta, A. (2015). Relación arroyo-acuífero en un sistema hídrico afectado por explotación antrópica. Estudios Ambientales 3 (2): 30-51.

Barranquero, R. S., Varni, M. R., Pardo, R., Vega, M., Zabala, M. E. y Ruiz de Galarreta, V. A. (2016a). Propuesta para el estudio comparativo del recurso hídrico subterráneo. Ejemplo de caso: cuencas de los arroyos Langueyú y Del Azul. Geología Aplicada a la Ingeniería y al Ambiente 37: 45-56. 
Barranquero, R. S., Varni, M. R., Pardo, R., Vega, M., Zabala, M. A. y Ruiz de Galarreta, V. A. (2016b). Joint interpretation of the hydrochemistry of two neighbouring basins by $\mathrm{N}$-way multivariate methods. Environmental Earth Sciences 75 (4): 335 1-14.

Bidaure, A. D. (2016). Diagnóstico ambiental del uso recreativo del arroyo Chapaleofú en la localidad de Rauch: pautas de gestión sustentable. (Tesis de Licenciatura en Diagnóstico y Gestión Ambiental). Universidad Nacional del Centro de la Província de Buenos Aires, Tandil.

Bidaure, A. D., Cortelezzi, A., Barranquero, R. S., Sganga, J. y Tabera, A. (2016). Evaluación del estado ecológico de un arroyo pampeano con uso recreativo.En: Ulberich A., Cisneros Basualdo N. y Miranda del Fresno C. (Eds). Actas I Jornadas Internacionales de Ambiente y III Jornadas Nacionales de Ambiente 2016. pp. 424-426. Tandil, Argentina: Universidad Nacional del Centro de la Provincia de Buenos Aires.

Bogetti, L. (2015). Diagnóstico ambiental de la Cuenca Superior del Arroyo Langueyú, Tandil. (Tesis de Licenciatura en Diagnóstico y Gestión Ambiental). Universidad Nacional del Centro de la Província de Buenos Aires, Tandil.

Cap-Net (2008). Conflict resolution and negotiation skills for Integrated Water Resources Management. Training manual. Recuperado de: http://www.capnet.org/sites/cap-net.org/files/Conflict\%20Manual\%20Final\%20170908.pdf

Cifuentes, M. (2012). Algas cianofitas y aptitud recreativa del Lago del Fuerte (Partido de Tandil). (Tesis de Licenciatura en Diagnóstico y Gestión Ambiental). Universidad Nacional del Centro de la Província de Buenos Aires, Tandil.

Cifuentes, M R., Gabellone, N A., Ruiz de Galarreta, A. (2017). Caracterización fisicoquímica de las aguas superficiales en la cuenca del arroyo Del Fuerte, Tandil. En: VI Congreso Bianual PROIMCA - IV Congreso Bianual PRODECA. Bahía Blanca, Argentina.

Cortelezzi, A. (2010). Hábitats funcionales y macroinvertebrados en cauces modificados de arroyos de llanura: impacto sobre la calidad ecológica. (Tesis de grado). Universidad Nacional de La Plata, La Plata. 
Custodio, E., M. Manzano y C. Montes. (2008). Perspectiva general del papel y gestión de las aguas subterráneas en el área de Doñana, Sudoeste de España. Boletín geológico y minero, 119 (1): 81-92.

Díaz, A. (2010). Evaluación y propuesta de gestión hídrica sanitaria en el Paraje De La Canal, Partido de Tandil. (Tesis de Maestría en Ingeniería Ambiental). Universidad Tecnológica Nacional, La Plata.

Duverger, M. (1981). Método de las ciencias sociales. Ed. Ariel, Barcelona.

García, R. (2011). Interdisciplinariedad y sistemas complejos. Revista Latinoamericana de Metodología de las Ciencias Sociales, 1 (1): 65-101.

Gómez Orea, D. y Gómez Villarino, A. (2012). Ordenamiento territorial: una aproximación conceptual y su aplicación al cantón Cuenca, Ecuador. En: Revista Universidad Verdad, № 57 Ordenamiento Territorial. p. 29-88.

Gutiérrez, R. A. (2010). Modelo para armar: gestión del agua en la provincia de Buenos Aires. En: Isuani, F. (Ed.). Política pública y gestión del agua: aportes para un debate necesario. pp. 153-196. Buenos Aires: Prometeo-UNGS.

GWP - Global Water Partnership (2009). Manual para la gestión integrada de Recursos Hídricos en Cuencas. Recuperado de: http://www.gwptoolbox.org/images/stories/Docs/handbook\%20inbo gwp spani sh.pdf

IHLLA - Instituto de Hidrología de Llanuras. (1995). Diagnóstico limnológico, sanitario, ictiológico y estado ambiental del Lago del Fuerte, ciudad de Tandil. Informe final para la Municipalidad de Tandil. $160 \mathrm{p}$.

IHLLA - Instituto de Hidrología de Llanuras. (2005a). Estudio de la calidad del agua del Lago del Fuerte, Tandil. Diagnóstico. Informe № 3 para la Municipalidad de Tandil. $17 \mathrm{p}$.

IHLLA - Instituto de Hidrología de Llanuras. (2005b). Monitoreo de Toxinas algales (microcistinas). Lago del Fuerte, Tandil. Informe Final para la Municipalidad de Tandil. $15 \mathrm{p}$.

Kennedy, K.; Simonovic, S.; Tejada-Guibert, A.; de França Doria, M.; Martin, J. L. (2009). IWRM Implementation in basins, sub-basins and aquifers: state of the art review. International Hydrological Programme of UNESCO. París. Recuperado de: http://unesdoc.unesco.org/images/0018/001817/181790e.pdf 
Peluso, F; Usunoff, E; González Castelain, J y Grosman, F. (1996). Management alternatives to improve the environmental quality at Del Fuerte Lake, Argentina. Part I. Methodological issues and Part II. Results and proposal. Journal of Environmental Hydrology. Vol. 4. Recuperado de: http://www.hydroweb.com/jeh/jeh1996/usunoff.pdf

Pessolano, B. (2011). Análisis geohidrológico de la cuenca del arroyo Chapaleofú Chico, Tandil, y su relación con las prácticas agropecuarias. (Tesis de Licenciatura en Diagnóstico y Gestión Ambiental). Universidad Nacional del Centro de la Província de Buenos Aires, Tandil.

Pujadas, R. y Font, J. (1998). Ordenación y planificación territorial. Madrid, España: Editorial Síntesis, Colección Espacios y Sociedades.

Rodriguez, C. I. (2010). Evaluación ambiental de las condiciones de explotación y uso del recurso hídrico subterráneo en el barrio Cerro Los Leones, Tandil. (Tesis de la Maestría en Evaluación Ambiental de Sistemas Hidrológicos). Universidad Nacional de La Plata, La Plata.

Rodriguez, C. I.; Jacinto, G. P.; Ruiz de Galarreta, V. A.; Banda Noriega, R. B. (2010). Gestión del agua subterránea en el Barrio Cerro Los Leones, Tandil (Argentina). Revista Ciencia Docencia y Tecnología, Vol. 41: 193-216.

Rodríguez, C. I.; Miguel, R. E.; André, M. J.; García, M. C.; Ruiz de Galarreta, A.; Banda Noriega, R. (2011). Análisis del agua subterránea en la localidad de Gardey (Tandil, Buenos Aires). En: García, R. y Rocha Fasola, V. (Eds.). Calidad y contaminación del agua subterránea. Actas del VII Congreso Argentino de Hidrogeología y $\mathrm{V}$ Seminario Hispano Latinoamericano sobre temas actuales de Hidrología subterránea. pp. 132-139. Salta, Argentina.

Rodriguez, C. I.; Miranda del Fresno, M.C.; Miguel, R. E.; Ulberich, A. C.; Ruiz de Galarreta, A. (2013a). Cambios de uso del suelo e impactos sobre el agua subterránea en un barrio al sur de Tandil, Buenos Aires, Argentina. Revista AUGM Domus, Vol. 5: 75-90.

Rodriguez, C. I.; Díaz, A.; Jacinto, G.; Tabera, A.; Pessolano, B.; Ruiz de Galarreta, A. (2013b). Evaluación del agua subterránea y transformaciones territoriales en María Ignacia-Vela, Tandil (Buenos Aires, Argentina). En: González N., Kruse E., Trovatto M. M. y Laurencena P. (Eds.). Agua subterránea recurso 
estratégico. Actas del VIII Congreso Argentino de Hidrogeología, Tomo I, pp. 306-313. La Plata, Argentina: Editorial EDULP.

Rodríguez, C. I. (2014). Evaluación ambiental del uso y gestión del agua subterránea en el partido de Tandil. Pautas para su gestión sustentable. (Tesis del Doctorado en Ciencias Naturales). Universidad Nacional de La Plata, La Plata.

Rodríguez, C. I. y Jacinto, G. P. (2017). Recursos hídricos: calidad y procesos de gestión en la región pampeana argentina. Revista Luna Azul № 44: 59-79.

Rodrigues Capítulo, A., M. Tangorra y C. Ocón. (2001). Use of benthic macroinvertebrates to assess the biological status of Pampean streams in Argentina. Aquatic Ecology 35 (2): 109-119.

Ruiz de Galarreta, A. y Banda Noriega, R. (2005). Geohidrología y evaluación de nitratos del Partido de Tandil, Buenos Aires, Argentina. En: IV Congreso Argentino de Hidrogeología y II Seminario Hispano-Latinoamericano sobre temas actuales de la Hidrología Subterránea, pp. 99-108. Río Cuarto, Argentina.

Ruiz de Galarreta, V. A. (2006). Geohidrología y balance hidrológico de la zona no saturada en la cuenca superior del arroyo Tandileofú, Provincia de Buenos Aires. (Tesis del Doctorado en Ciencias Naturales). Universidad Nacional de La Plata, La Plata.

Ruiz de Galarreta, V. A.; Banda Noriega, R. B.; Barranquero, R. S.; Díaz, A. A.; Rodríguez, C. I.; Miguel, R. E. (2010). Análisis integral del sistema hídrico, uso y gestión. Cuenca del arroyo Langueyú, Tandil, Argentina. Boletín Geológico y Minero. 121 (4): 343-356.

Ruiz de Galarreta, A. y Varni, M. (2010). Análisis de la recarga del acuífero freático en la cuenca superior del arroyo Tandileofú. Partido de Tandil. En: Ulberich, A. (Coord). Estudios Ambientales II "Tandil y área de influencia". pp. 81-90. Tandil, Argentina: Universidad Nacional del Centro de la Provincia de Buenos Aires.

Ruiz de Galarreta, A.; Barranquero, R.; Varni, M. y Rodriguez, C. I. (2011). Geología e hidrolitología de la cuenca del Arroyo Langueyú, Provincia de Buenos Aires (Argentina). Revista Ciencia Docencia y Tecnología. Vol. 43: 157-176. 
Ruiz de Galarreta, A.; Banda Noriega, R.; Najle, R.; Rodriguez, C.; Barranquero, R.; Díaz, A.; Miguel, E.; Pereyra, M. y Priano, M. (2013). Análisis de la calidad del agua del arroyo Langueyú, Tandil, Buenos Aires. Revista Estudios Ambientales. Vol. 1 № 1: 2-26. 